\title{
Yes to Success: a Guide to Taking Control of Your Career and Time
}

\author{
Marley Doyle ${ }^{1}$
}

Received: 28 February 2020 / Accepted: 20 May 2020 /Published online: 29 May 2020

(C) Academic Psychiatry 2020

Early-career physicians in academic medicine leave their institutions at higher rates than mid- to late-career physicians [1]. To retain and support faculty throughout their careers, we need to consider factors that may contribute to the departure. Many early-career faculty struggle to find their identity and manage the expected commitments in clinical work, research, education, and service. In a qualitative study of new family medicine faculty at one institution [2], the faculty identified three critical career concerns: lack of or inadequate orientation, the challenges associated with transitioning to academia, and balancing the demands of the role. Early-career faculty attempt to navigate a complicated and ever-changing system with little to no mentorship on how to steer the ship. Identification of a career mentor during orientation could help with some of these concerns.

The hierarchical culture of academic medicine may be partly to blame for this oversight. We teach medical students and residents to express gratitude and say yes to any project offered by a faculty member. The advice can be direct, but often, colleagues and mentors model from examples. One of my mentors once advised me to "Say yes to everything" as a trainee and early-career physician. The unspoken message I inferred was that my success depended on my compliance with requests. I took this advice and quickly found that I was overstretched and unfocused. Although my schedule was hectic, I had few promotable activities on my curriculum vitae. I was well trained to say yes but lacked the skills to know how or when to say no. Consequently, I questioned the advice, and I now operate under a different philosophy: Say yes to success and no to woe. For this philosophy, I modified the reporters' questions (i.e., Who? What? When? Where? Why? How?) as a tool to help me make decisions. Using these simple

Marley Doyle

Marley.Doyle@unmc.edu

1 University of Nebraska Medical Center, Omaha, NE, USA questions before committing to any new commitment allows more attention and energy to promotable activities that align with your priorities.

\section{Who Is Asking?}

Consider who is asking: Your supervisor? A colleague? A medical student? Think about the implications of your answer. For example, it may be unwise to say no to your supervisor or to say no to anything related to your job description. You may have more flexibility in other circumstances, however. Do not be afraid to say "yes, but." This phrase can help you set the terms of the commitment to fit with your schedule. For instance, "Yes, but could you give me some more background?" (or time or support). A denied request may follow, but the additional information will aid in decision making.

\section{What Is the Time Commitment?}

You should always know the time commitment before saying yes. If asked to take a leadership position, ask about the term. Many responsibilities have indefinite term limits, so the time commitment is something you want to discuss before agreeing. If joining a committee or board, ask how often the committee meets and if you would have other responsibilities outside of the meetings.

\section{When Will I Do This?}

Once you know the time commitment, it is essential to reflect on when you will do the activity. I am a big proponent of scheduling everything into my calendar, which allows me to visualize where the commitment could fit. Aside from a work calendar, other responsibilities need to be considered, including the responsibility to your wellness. 


\section{Where Does This Fit into My Career Goals and Values?}

Early on, it is difficult to know what your path is. Saying yes can open up opportunities and help define your career path. If you are a few years out of training, you may have your career goals clearly defined. It then becomes easier to evaluate whether an activity helps or hinders your advancement. If you are in academics, you can ask yourself, "Will this opportunity get me promoted?" If the answer is no, then you must have other reasons to do it. To do this evaluation, you need to know your values and priorities. If you find an activity energizing, enjoyable, and fulfilling, then say yes. Perhaps talking to a student interest group about a career in psychiatry will not lead to promotion, but if you love doing it, then you should.

\section{Why Me?}

Why were you asked over someone else? Is someone trying to help elevate your career? Sometimes people see strengths and potential that we may not see in ourselves. Perhaps someone chose you for a reason. However, if you get the sense that a "warm body" is needed, then you may want to consider carefully. The tension between fulfilling departmental and personal needs will always be present. Most people will serve in a minimally satisfying role at some point in their academic careers. These experiences can have value, but it helps if the position aligns with personal goals as much as possible.

\section{How Am I Going to Respond?}

Once you decide, a graceful response is imperative for either outcome. Be sure to thank the person for the recognition. If you say no, always make sure that you give a reason and nominate someone else. Be thoughtful about your goal and frame your decision in the context of departmental or institutional needs. When in doubt, ask a mentor or trusted friend to help you navigate the decision.

This system has helped me develop a career focus, and I find that my work is more satisfying and purposeful. This system is not perfect. I am still overcommitted, but now I focus on activities that are important to me. My only regret is that I did not have these skills earlier. In my opinion, these skills would be most beneficial in training but also acceptable in new faculty orientation. Perhaps if more physicians had these skills, fewer would leave academic medicine, which would lead to a more diverse, inclusive, and fulfilled workforce.

\section{Compliance with Ethical Standards}

Disclosures The author states that there is no conflict of interest.

\section{References}

1. Kubiak NT, Roman J, Guidot DM, Franklin Trimm R, Kamen DL. Recruitment and retention in academic medicine - what junior faculty and trainees want department chairs to know. Am J Med Sci. 2012;344(1):24-7.

2. Levy M, Koppula S, Brown JB. Transitioning to academia: exploring the experience of new family medicine faculty members at the beginning of their academic careers. Can Fam Physician. 2018;64(12):907-13.

Publisher's Note Springer Nature remains neutral with regard to jurisdictional claims in published maps and institutional affiliations. 